\title{
Breaking the mass/anisotropy degeneracy in the Coma cluster
}

\author{
Gary A. Mamon ${ }^{1}$, Ewa L. Lokas $^{2}$ and Teresa Sanchis ${ }^{3}$ \\ ${ }^{1}$ IAP, Paris, France email: gam@iap.fr \\ ${ }^{2}$ Copernicus Center, Warsaw, Poland email: lokas@camk.edu.pl \\ ${ }^{3}$ Dep. di Astronomia i Meteorologia, Univ. de Barcelona, Spain email: tsanchis@am.ub.es
}

\begin{abstract}
We provide the first direct lifting of the mass/anisotropy degeneracy for a cluster of galaxies, by jointly fitting the line of sight velocity dispersion and kurtosis profiles of the Coma cluster, assuming an NFW tracer density profile, a generalized-NFW dark matter profile and a constant anisotropy profile. We find that the orbits in Coma must be quasi-isotropic, and find a mass consistent with previous analyses, but a concentration parameter $50 \%$ higher than expected in cosmological $N$-body simulations. We then test the accuracy of our method on realistic non-spherical systems with substructure and streaming motions, by applying it to the ten most massive structures in a cosmological $N$-body simulation. We find that our method yields fairly accurate results on average (within 20\%), although with a wide variation (factor 1.7 at $1 \sigma$ ) for the concentration parameter, with decreased accuracy and efficiency when the projected mean velocity is not constant with radius.
\end{abstract}

\section{Introduction}

The kinematical ("Jeans") analyses of near-spherical structures using the spherical Jeans equation for stationary systems:

$$
\frac{\mathrm{d}}{\mathrm{d} r}\left(\nu \sigma_{r}^{2}\right)+\frac{2 \beta}{r} \nu \sigma_{r}^{2}=-\nu \frac{G M}{r^{2}},
$$

where $\nu$ is the 3D density distribution of the tracer population, suffer from the fact that this one equation involves two unknowns: the mass distribution $M(r)$ and the velocity anisotropy $\beta=1-\sigma_{\theta}^{2} / \sigma_{r}^{2}$ (we make use of the symmetry of spherical systems yielding $\left.\sigma_{\phi}=\sigma_{\theta}\right)$. In some cases of simple anisotropy profiles $\beta(r)$, the Jeans equation can be inverted to yield the radial dispersion as a single integral over radii of $\nu M$ times some kernel, and one can insert this solution into the equation that links the line-ofsight velocity dispersion to the radial one, to obtain after some algebra, the line-of-sight dispersion as a single integral over $\nu M$ and some other kernel.

In what follows, we attempt to lift the mass/anisotropy degeneracy by adding a second equation, namely the 4 th order Jeans equation for constant anisotropy (Lokas 2002)

$$
\frac{\mathrm{d}}{\mathrm{d} r}\left(\nu \overline{v_{r}^{4}}\right)+\frac{2 \beta}{r} \nu \overline{v_{r}^{4}}+3 \nu \sigma_{r}^{2} \frac{\mathrm{d} \Phi}{\mathrm{d} r}=0
$$

where we express the line of sight kurtosis as a double integral over $\nu M$ and $M$ and a third kernel (the details are given in Łokas \& Mamon 2003).

\section{Kinematic analysis of the Coma cluster}

We apply our formalism to the nearby Coma cluster, for which we've extracted from NED the positions and line-of-sight velocities of 967 galaxies, among which 355 early-type 

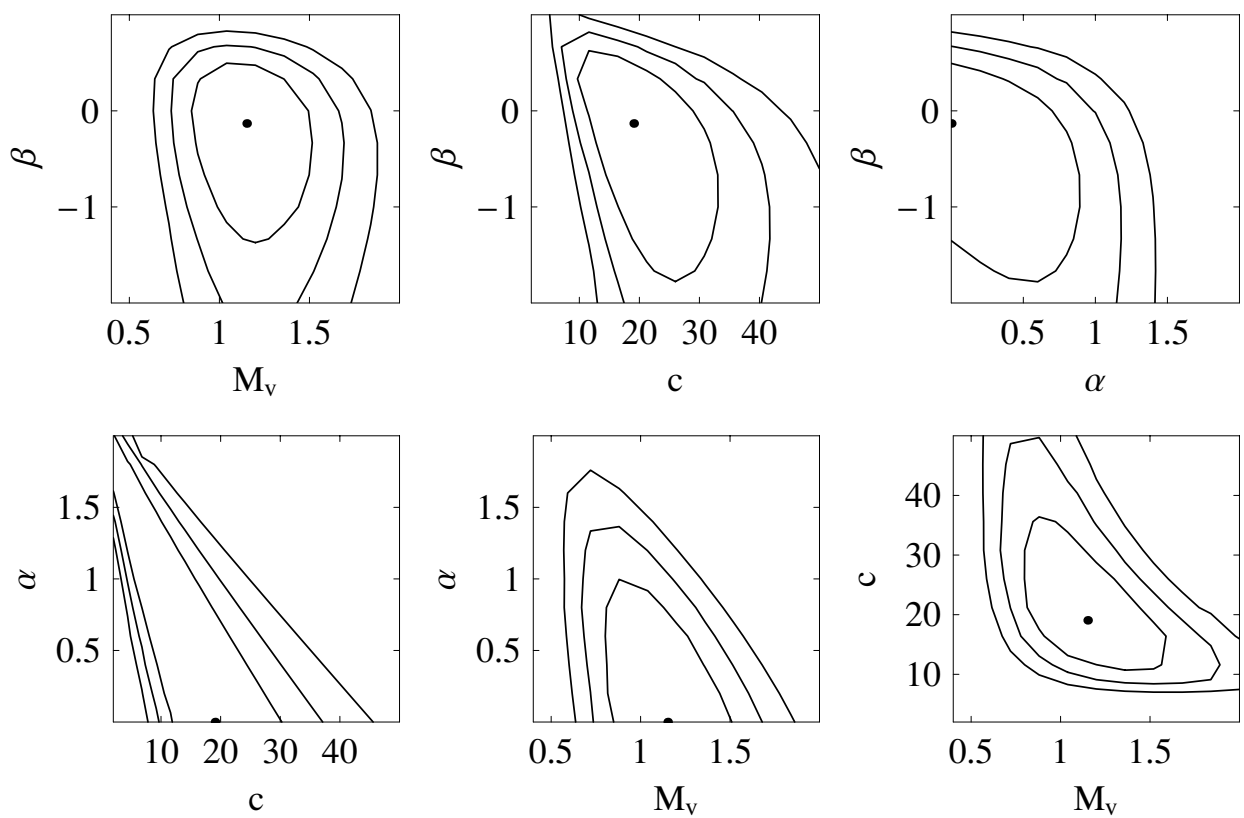

Figure 1. Probability contours $(1,2$, and $3 \sigma)$ for joint fits of $\sigma_{\operatorname{los}}$ and $\left(\log \kappa_{\operatorname{los}}\right)^{1 / 10}$ (the two are found to be independent). Circles indicate the best-fitting parameters. The mass is in units of $10^{15} M_{\odot}$.

galaxies whose kinematics we analyze here (we omit spiral galaxies, which are expected to have a non negligible mean radial velocity caused by their infall and subsequent transformation at cluster pericenter). Our mass model includes 1) a stellar component, which we find is well fit by an NFW (Navarro et al. 1996) model; 2) a dark matter component, which we assume to be a generalized NFW model with arbitrary inner slope:

$$
\rho(r) \propto(r / a)^{-\alpha}(1+r / a)^{\alpha-3},
$$

where $a$ is the scale radius of the dark matter (in general different from that of the luminous distribution), and $\alpha=1$ for the NFW model. The profiles differ by their inner slope $-\alpha$ but have a common outer limiting behavior of $r^{-3}$; and 3) an isothermal gas component, taken from the ROSAT X-ray observations by Briel et al. (1992).

Using 7 radial bins at $R<80^{\prime}$, we jointly fit our four parameters, mass $M_{v}$ within the virial radius $r_{v}$, velocity anisotropy $\beta$, inner slope $\alpha$ of the dark matter density profile, and dark matter concentration $c=r_{v} / a$. Contrary to the case when only velocity dispersion is studied, the minimization procedure now converges (except for a concentration / inner slope degeneracy). The best fits are shown in Figure 1. The best fit parameters are $M_{v}=1.2 \times 10^{15} M_{\odot}$ (corresponding to dark matter virial radius $r_{v}=92^{\prime}=2.7 \mathrm{Mpc}$ ), $\beta=-0.13, \alpha=0$ and $c=19$ with $\chi^{2} / N=6.1 / 10$. In other words, our best fit models are isotropic and the generalized dark matter model of equation (2.1) provides excellent fits to the data.

Our best fit radial profiles for each half-integer value of $\alpha$ are shown in Figure 2 . The best fit density profiles are indistinguishable beyond $0.03 r_{v}$, and the outer mass profiles are very close to the infall estimates of Geller, Diaferio \& Kurtz (1999). The density profiles are $50 \%$ more concentrated than in structures of comparable mass within 

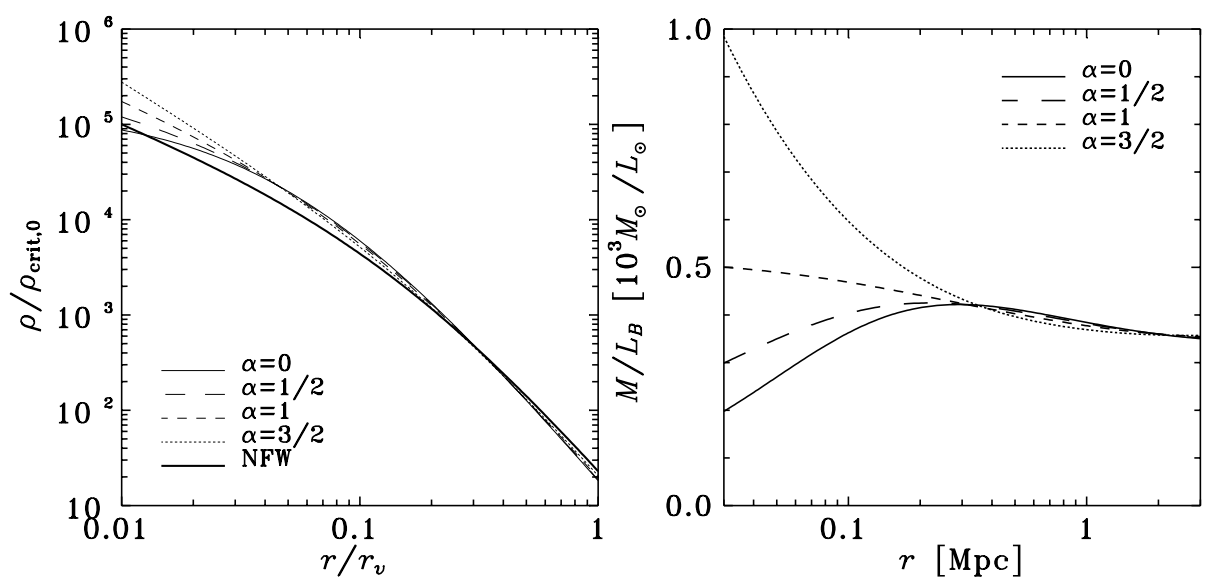

Figure 2. Best fitting dark matter profiles with different inner slopes (left panel) and their associated mass-to-light ratios (right panel), for different $\alpha$ increasing upwards from 0 to 1.5 (with $c=19,14,9$ and 5 , respectively). The dark matter virial radii are $r_{v}=2.7 \mathrm{Mpc}$ for all models shown. The heavy solid curve in the left plot indicates the best NFW model $(c=6)$ from cosmological $N$-body simulations, which is still (marginally) consistent with the data.

cosmological $N$-body simulations in a $\Lambda$ CDM Universe (Bullock et al. 2001). Interestingly, the $M / L$ profiles have nearly constant slope for the NFW case $(\alpha=1)$.

Our analysis differs from previous kinematical analyses (e.g. Merritt 1987; den Hartog \& Katgert 1996; Carlberg et al. 1997; van der Marel et al. 2000; Biviano \& Girardi 2003; Katgert et al. 2004), as 1) we have, for a single cluster, a larger sample of galaxies, which, given their early morphological type, should be in dynamical equilibrium in the cluster potential; 2) we remove pairs from the computation of the velocity moments; 3 ) we include kurtosis in the analysis; 4) we model the dark matter distribution using a generalized formula inspired by the results of cosmological $N$-body simulations; 5 ) we include hot gas.

In comparison to studies based upon stacking of many clusters, our analysis of the Coma cluster benefits from not having to introduce errors in any stacking procedure, and from a cleaner removal of interlopers. On the other hand, the analyses of stacked clusters have the advantage of averaging out particular inhomogeneities of individual clusters such as Coma, expected in hierarchical scenarios of structure formation and observed in Coma, both in projected space (Fitchett \& Webster 1987; Mellier et al. 1988; Briel et al. 1992) and velocity space (Colless \& Dunn 1996; Biviano et al. 1996).

\section{Kinematic analysis of simulated clusters}

In our Jeans analysis of the Coma cluster, we assumed that the cluster was spherical, with no substructure nor streaming motions, and that projection effects were not serious. We test (details in Sanchis, Łokas \& Mamon 2004) these assumptions using a $\Lambda$ CDM cosmological $N$-body (tree-code) simulation, run by Ninin (1999), with $256^{3}$ particles, and with $\Omega_{m}=1 / 3, \Omega_{\lambda}=2 / 3, h=2 / 3$, and $\sigma_{8}=0.88$ (details in Hatton et al. 2003).

We analyze each of the 10 most massive halos along 3 axes: the major axis, an orthogonal one and an intermediate one. Figure 3 shows the radial profiles of line-of-sight velocity moments (mean, dispersion, skewness and kurtosis) along the principal axis. These profiles display statistically significant radial variations caused by substructures 

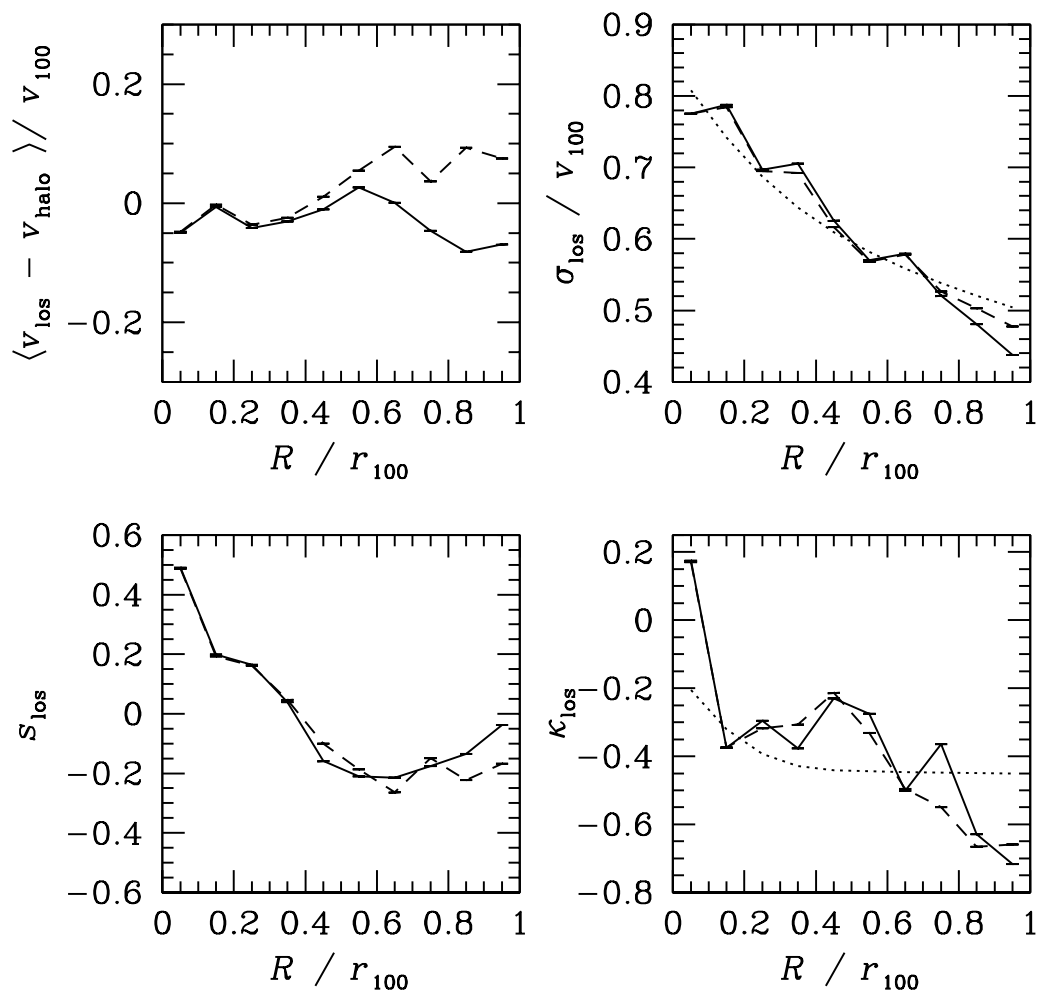

Figure 3. Projected velocity moments of the dark matter particles in halo 1 measured along the principal axis. The upper left panel shows the mean line-of-sight velocity with respect to the velocity of the center of the halo in units of the circular velocity at $r_{100}$. The upper right panel gives the line-of-sight velocity dispersion in the same units. The two lower panels give the skewness (left) and kurtosis (right). In each panel the solid line shows results for particles lying inside the sphere of radius $r_{100}$, while the dashed line is for all particles. The dotted curve shows the fits obtained from the Jeans equations.
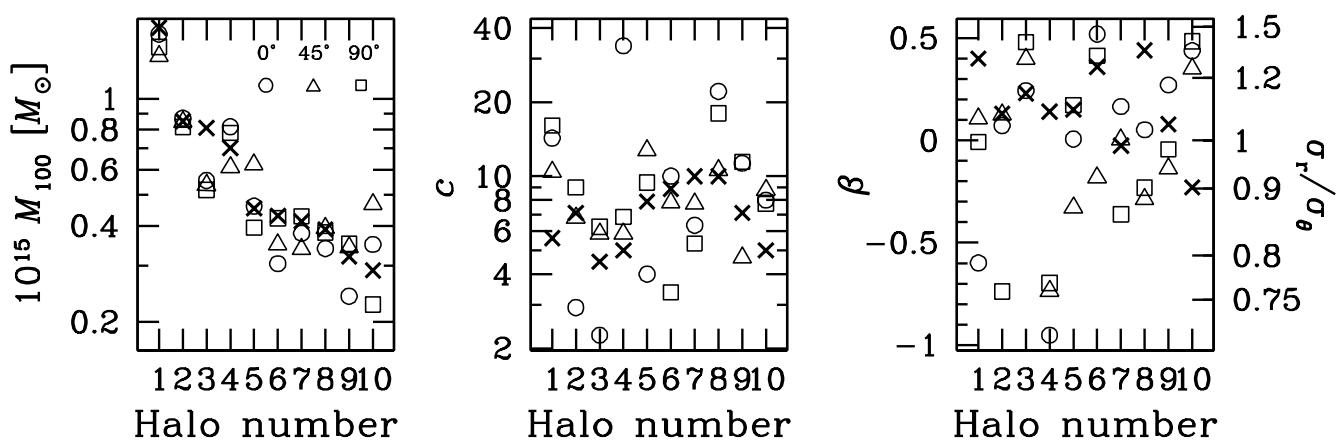

Figure 4. Fitted (on projected data) values (using 40 particles per bin) of virial mass $M_{100}$, concentration parameter $c$ and anisotropy $\beta$ of the ten halos for the three directions of observation with respect to the major axis of each halo: $0^{\circ}$ (circles), $45^{\circ}$ (triangles) and $90^{\circ}$ (squares). The values measured on 3D data are shown with crosses. 


\begin{tabular}{|c|c|c|c|c|c|c|c|c|}
\hline \multirow{2}{*}{$\begin{array}{l}\text { Particles } \\
\text { per bin }\end{array}$} & \multicolumn{2}{|c|}{$\Delta \log M_{100}$} & \multicolumn{2}{|c|}{$\Delta \log c$} & \multicolumn{2}{|c|}{$\Delta \beta$} & \multicolumn{2}{|c|}{$\Delta \log \left(\sigma_{r} / \sigma_{\theta}\right)$} \\
\hline & mean & $\sigma$ & mean & $\sigma$ & mean & $\sigma$ & mean & $\sigma$ \\
\hline A & -0.03 & 0.09 & 0.20 & 0.18 & -0.78 & 1.04 & -0.12 & 0.12 \\
\hline 40 & -0.07 & 0.10 & 0.08 & 0.24 & -0.20 & 0.48 & -0.04 & 0.11 \\
\hline
\end{tabular}

Table 1. Results of the fitting procedure

with radial streaming motions. Moreover, the moments vary considerably from halo to halo and for the 3 different projections of a given halo. Note also that the projected mean velocity differs substantially between all the particles in the projected cylinder and the subset within the virial sphere.

Figure 4 shows the results of our fits from our Jeans analysis (open symbols) in comparison with our fits to the $3 \mathrm{D}$ data (crosses) and Table 1 summarizes the statistical accuracy of our fitting procedure. The virial mass is typically underestimated by $15 \pm 26 \%$, while the concentration parameter is overestimated by $20 \pm 74 \%$ and the ratio $\sigma_{r} / \sigma_{\theta}$ underestimated by $9 \% \pm 29 \%$. Hence, our kinematical analysis yields good results on average, but with a fairly large dispersion in accuracies (with the most inaccurate estimates for halos with non zero mean line of sight velocities at some radii). Halo 4, which departs the most from the 3D measures of concentration and anisotropy, happens to have important variations in its projected mean velocity profile, whereas nearly all other halos have normal mean velocity profiles. This suggests that the joint dispersion/kurtosis Jeans analysis is adequate for structures with fairly constant projected mean velocity profiles. Given the large dispersion on the concentration parameter, our high value of $c$ for Coma is now consistent with the 1.5 times lower value expected from cosmological $N$-body simulations.

\section{References}

Biviano, A. et al. 1996 A $\& A$ 311, 95-112

Biviano, A. \& Girardi, M. 2003 ApJ 585, 205-214

Briel, U., Henry, J. P. \& Böhringer, H. 1992 A $\&$ A 259, L31-L34

Bullock, J. S. et al. 2001 MNRAS 321, 559-575

Carlberg, R. G. et al. 1997 ApJ 485, L13-L16

Colless, M. \& Dunn, A. M. 1996 ApJ 458, 435-454

den Hartog, R. \& Katgert, P. 1996 MNRAS 279, 349-388

Fitchett, M. \& Webster, R. 1987 ApJ 317, 653-667

Geller, M. J., Diaferio, A. \& Kurtz, M. J. 1999 ApJ 517, L23-L26

Hatton, S. et al. 2003 MNRAS 343, 75-106

Katgert, P., Biviano, A. \& Mazure, A. 2004 ApJ 600, 657-669

Łokas, E. L. $2002 M N R A S$ 333, 697-708

Łokas, E. L. \& Mamon, G. A. 2003 MNRAS 343, 401-412

Mellier et al. $1988 A \& A$ 199, 67-72

Merritt 1987 ApJ 313, 121-135

Navarro, J. F., Frenk, C. S. \& White, S. D. M. 1996 ApJ 462, 563-575

Ninin, S. 1999 PhD thesis, Univ. of Paris 11

Sanchis, T., Łokas, E. L. \& Mamon, G. A. 2004 MNRAS 347, 1198-1207

van der Marel, R. P. et al. 2000 AJ 119, 2038-2052 